\title{
Dependence of Ultrasonic and Magnetic Hyperthermia on the Concentration of Magnetic Nanoparticles
}

\author{
K. KaczmareK ${ }^{a}$, T. Hornowski ${ }^{a}$, R. Bielas ${ }^{a}$, D. ŻAK ${ }^{a}$, M. Timko ${ }^{b}$ And A. JózefczAK ${ }^{a, *}$ \\ ${ }^{a}$ Institute of Acoustics, Faculty of Physics, Adam Mickiewicz University, Umultowska 85, 61-614 Poznań, Poland \\ ${ }^{b}$ Institute of Experimental Physics, Slovak Academy of Sciences, Watsonova 47, 04001 Košice, Slovakia
}

\begin{abstract}
Hyperthermia treatment is the heating of tumor tissue up to temperatures between $41{ }^{\circ} \mathrm{C}$ and $45^{\circ} \mathrm{C}$, which trigger several physiological reactions in the body. Hyperthermia within tissue can be applied through various mechanisms. One of them is magnetic hyperthermia which uses superparamagnetic iron oxide nanoparticles (SPIONs) heated by an externally applied magnetic field. SPIONs can also be used as sonosensitizers in ultrasound hyperthermia increasing acoustic wave attenuation. The impact of SPION concentration on thermal effect during ultrasonic and magnetic hyperthermia was investigated in agar-gel phantom with added magnetite nanoparticles. The presence of nanoparticles in the tissue-mimicking phantom increases the thermal losses of ultrasound energy and temperature of the phantom.
\end{abstract}

DOI: 10.12693/APhysPolA.133.716

PACS/topics: 75.75.-c, 43.35.Cg, 43.35.Wa, 87.85.-d

\section{Introduction}

Hyperthermia is a very promising anti-cancer medical treatment. It induces heat in cancer tissues which leads to their weakening. Weaken cells are therefore more susceptible for other cancer therapies like radiotherapy or chemotherapy $[1,2]$. Heat in tissues can be induced by means of ultrasonic waves, alternating magnetic field, radiowaves or microwaves [3]. To be more specific, heating in the tumor cells is achieved through the absorption of ultrasonic waves or magnetic energy. However, satisfying results of hyperthermia treatment often demand application of high energies which can lead to unpleasant side effects like pain or skin burns [4]. Still, effectiveness of hyperthermia treatments can be improved without usage of high energies.

Many research shows that thermal effect of ultrasonic hyperthermia can be increased by employing scattering materials such as gold, silicone or magnetite nanoparticles. Additional scattering caused by these so-called sonosensitizers becomes the source of supplementary ultrasound attenuation which consequently leads to increase in temperature [5-7].

Clinically oriented hyperthermia experiments are conducted on tissue-mimicking phantoms which are sufficient equivalents for human tissues [8]. In our study, the agar phantoms were used to investigate the impact of magnetic nanoparticles concentration in phantoms on temperature rise during ultrasonic and magnetic hyperthermia.

\section{Sample preparation and measurement setups}

Two types of phantom were prepared for our measurements: pure agar-gel sample without any scattering material and agar-gel samples with magnetic nanoparticles.

\footnotetext{
*corresponding author; e-mail: aras@amu.edu.pl
}

Agar powder was dissolved in hot distilled water. During the preparation process of the agar-gel, the sonosensitizers (magnetite nanoparticles) were added. Magnetite nanoparticles were purchased from Sigma-Aldrich Co. LLC. Basic properties of $\mathrm{Fe}_{3} \mathrm{O}_{4}$ particles are as follows: purity $-97 \%$, form - nanopowder, particle - spherical, size $-50-100 \mathrm{~nm}(\mathrm{SEM})$, surface area $>60 \mathrm{~m}^{2} / \mathrm{g}$, and bulk density $-0.84 \mathrm{~g} / \mathrm{mL}$. The dependence of magnetization on the magnetic field measured at the room temperature by SQUID magnetometer is shown in Fig. 1. Magnetite nanoparticles used in our study tend to small hysteresis which means that these samples are the mixtures of superparamagnetic and ferromagnetic particles. The saturation magnetization $M_{s}$ is $91 \mathrm{emu} / \mathrm{g}$. Samples were made by varying the weight concentration of $\mathrm{Fe}_{3} \mathrm{O}_{4}$ nanoparticles from $0.4-5 \%(\mathrm{w} / \mathrm{w})$ and concentration of agar from $5-8.5 \%(\mathrm{w} / \mathrm{w})$.

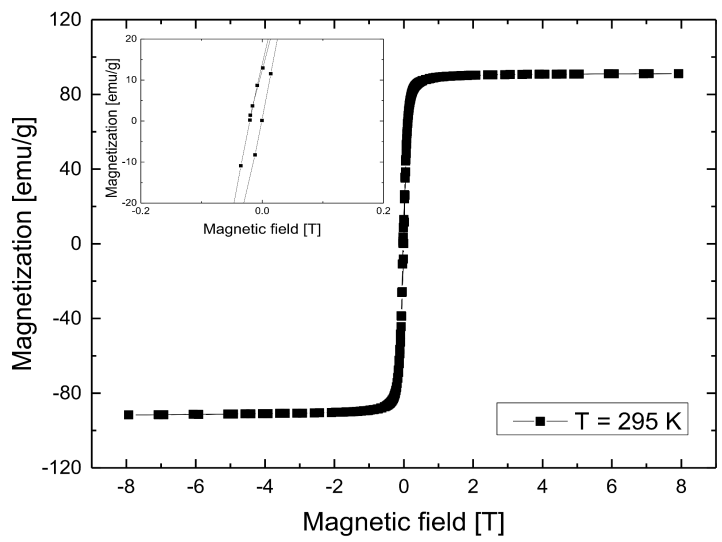

Fig. 1. Magnetization curve for the nanoparticles powder obtained from SQUID. The inset shows a small hysteresis.

The experimental setup for ultrasonic hyperthermia measurements is shown in Fig. 2(a). During experiments the measured sample was immersed in distilled water at 
room temperature. To avoid reflections from the plastic beaker in which sample was hold, the damping material was placed on the bottom of the beaker. Ultrasonic transducer with $1 \mathrm{~cm}^{2}$ diameter emitted $1 \mathrm{MHz}$ continuous ultrasonic wave. The whole sample was in near field of ultrasonic transducer which was about $1.2 \mathrm{~cm}$. The intensity of the ultrasonic beam was $2.5 \mathrm{~W} / \mathrm{cm}^{2}$. Figure 2(b) presents the experimental setup for magnetic hyperthermia measurements. The phantom in a glass tube was placed in the center of the $205 \mathrm{~mm}$ long magnetic coil with $n=56$ turns and radius $R=38.5 \mathrm{~mm}$. The frequency of alternating magnetic field was $180 \mathrm{kHz}$ and the intensity of the magnetic field, in the middle of the coil on-axis, was $5.5 \mathrm{kA} / \mathrm{m}$. The water cooling system for magnetic coil was maintained by PolyScience programmable temperature controller.

In all experiments temperature was measured with the optical thermometer Evolution FISO FPI-HR model FOT-L-SD. During ultrasonic hyperthermia the thermometer was centrally placed, i.e. $0.5 \mathrm{~cm}$ from the surface of the sample.

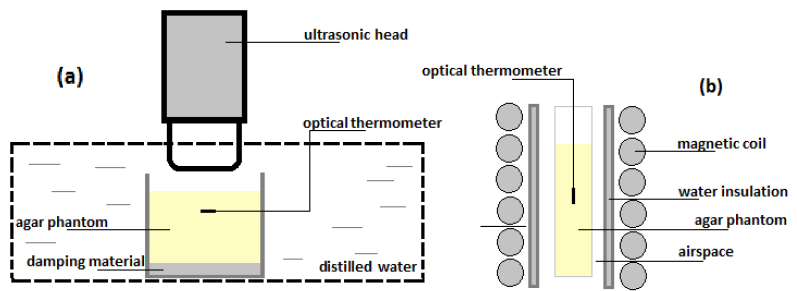

Fig. 2. Experimental setup for a) ultrasonic hyperthermia measurements, b) magnetic hyperthermia measurements.

\section{Experimental results}

In our study ultrasonic wave attenuation coefficient and thermal effect of ultrasonic and magnetic hyperthermia were measured in prepared samples. Figure 3 presents ultrasonic wave attenuation (measured by the pulse-echo technique for the wave of frequency $f=$ $5 \mathrm{MHz}[8]$ ) for agar phantoms with different concen-

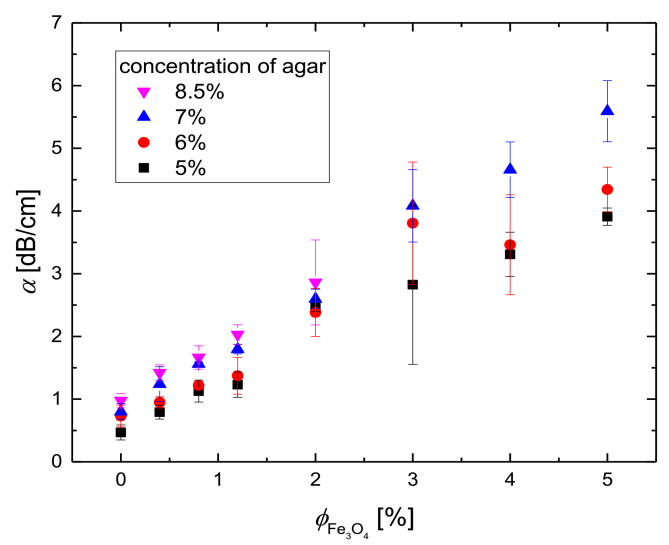

Fig. 3. Ultrasonic wave attenuation vs. concentration of magnetite nanoparticles in agar phantoms for different concentrations of agar in water $(5-8.5 \% \mathrm{w} / \mathrm{w})$.

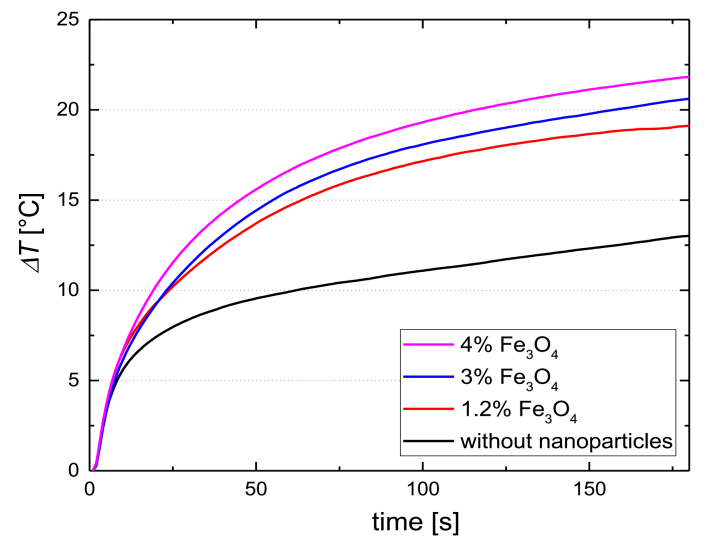

Fig. 4. Relative temperature rise with time in $5 \%$ agargel phantoms for different concentrations of magnetite nanoparticles during ultrasonic heating.

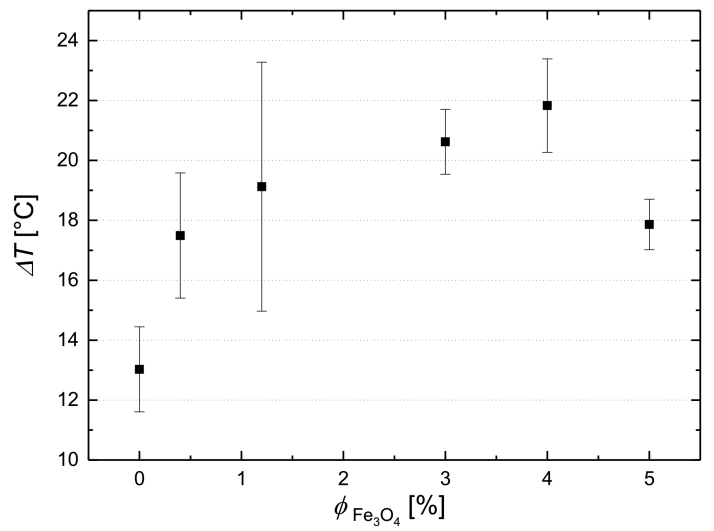

Fig. 5. Increase in temperature obtained during 180 seconds of ultrasonic sonication for $5 \%$ agar gel phantom with different concentration of magnetite nanoparticles.

tration of magnetite nanoparticles varying from $0.4-5 \%$ $(\mathrm{w} / \mathrm{w})$. A monotonic rise of attenuation coefficient with the increase of agar and magnetite concentration can be noticed. As it is seen in Fig. 3 the magnetite nanoparticles added to pure agar-gel phantoms induces the scattering of ultrasonic waves which leads to the additional attenuation. This in turn, becomes the source of the additional increase in temperature during ultrasonic hyperthermia. It means that the application of nanoparticles clearly improves the effectiveness of hyperthermia treatment. Figure 4 presents the relative temperature change with time for pure agar phantoms as well as agar phantoms with three different concentration of magnetite. Clearly, the temperature rise with increasing concentration of magnetic nanoparticles can be noticed. Figure 5 shows the increase in temperature after 180 seconds of ultrasonic sonication for $5 \%$ agar gel phantom with different concentrations of magnetite nanoparticles. The temperature rise due to ultrasonic heating increase with the amount of magnetic particles. A drop in temperature for $5 \%$ concentration of nanomaterial can be explained by high backscattering of ultrasound which is visible in 


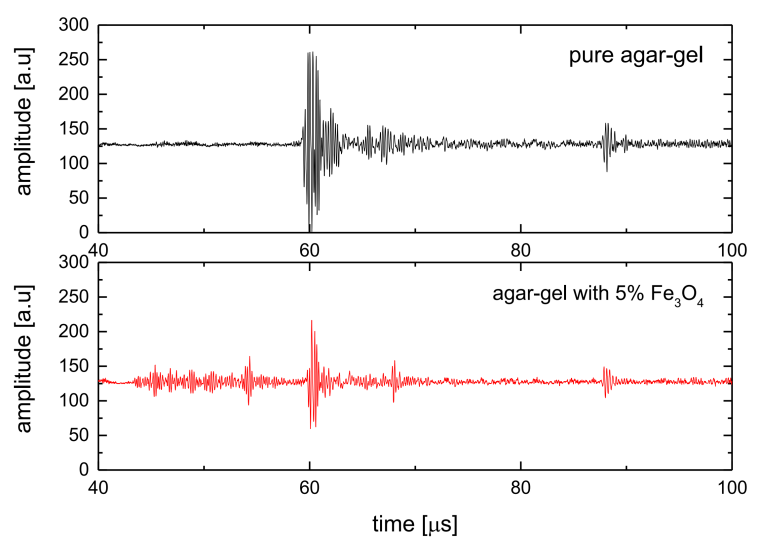

Fig. 6. Ultrasonic echo signals in pure-agar sample and agar sample with magnetite nanoparticles.

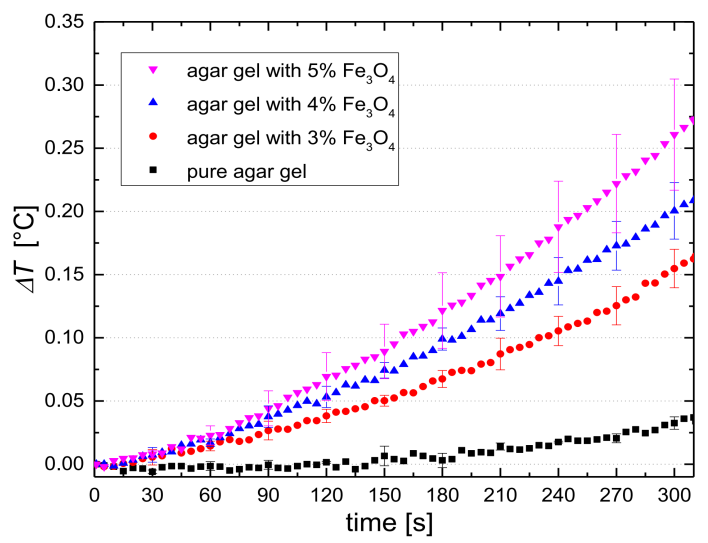

Fig. 7. Relative temperature rise vs. time for pure agar-gel samples and for agar-gel with different concentration of magnetite nanoparticles. Concentration of agar powder in all samples was $5 \%$.

Fig. 6 in the interval between $40^{\text {th }}$ and $60^{\text {th }} \mu \mathrm{s}$. Figure 7 presents the relative temperature change with time obtained during magnetic heating in AC magnetic field for pure agar-gel and for agar-gel with different concentrations of magnetite nanoparticles. In all samples the concentration of agar powder in water was $5 \%$ and concentration of magnetite in agar phantoms varied from $3-5 \%$ $(\mathrm{w} / \mathrm{w})$. Observed relative temperature change under the influence of alternating magnetic field also increases with the amount of nanoparticles. The obtained rise in temperature is rather small due to the quenching of Brown relaxation mechanism by the confinement of nanoparticles in agar-gel [7] so the only cause of magnetic heating is the Néel relaxation mechanism which strongly depends on the size of nanoparticles. In our previous work [7] we used samples with $0.8 \%(\mathrm{w} / \mathrm{w})$ concentration of nanoparticles with $10 \mathrm{~nm}$ mean size and the temperature rise during magnetic heating was better than in this study. This confirms that the size of magnetite nanoparticles is the crucial factor for the effectiveness of both ultrasound and magnetic heating. In the former large nanoparticles cause the ultrasound backscattering, while in the latter they extend the Néel relaxation time which is the only heating mechanism in situation when Brown relaxation is quenched due to the confinement of nanoparticles in agar-gel. In both cases the unsuitable size of sonosensitizer nanoparticles leads to deterioration in temperature rise.

\section{Conclusions}

The obtained experimental results confirm theoretical assumptions that the addition of scattering material enhances heat production by ultrasound wave in the tissuemimicking phantom due to increase of ultrasound attenuation. Magnetite nanoparticles can therefore be used as an effective sonosensitizers. On the other hand, magnetite nanoparticles are able to produce heat by themselves in the AC magnetic field which leads to further increase of temperature in tissue phantoms. The temperature rise obtained due to the addition of magnetite nanoparticles allow us to shorten the duration time of hyperthermia treatments and reduce the needed intensity of ultrasonic wave and the strength of magnetic field in the therapy. It was shown that, generally, the bigger amount of nanoparticles, the higher temperature can be obtained. However, the size and concentration of magnetite nanoparticles should be carefully considered because for higher concentration a possibility of aggregation may arise. In this case part of ultrasound wave energy instead of producing heat would be scattered outside the region of interest and the temperature rise would deteriorate. Furthermore, the presence of bigger nanoparticles in phantom disables the heating based on Néel relaxation leaving Brown relaxation as the only heating mechanism which is unfortunately inefficient when magnetic nanoparticles are confined in agar-gel.

Nevertheless, by merging magnetic and ultrasonic hyperthermia we can achieve a better heating of tumor tissue than in separate treatments [7]. So, this kind of synergistic therapy should be considered in the future.

\section{Acknowledgments}

This work was supported by a National Science Centre grant, Poland, no 2015/17/B/ST7/03566. M.T. acknowledges support via VEGA grants No. 2/0141/16 and $1 / 0311 / 15$ and Ministry of Education Agency for structural funds of EU Project No. 26220120003.

\section{References}

[1] B. Hildebrandt, et al., Crit. Rev. Oncol. Hematol. 43, 33 (2002).

[2] J.C. Peeken, et al., Front. Oncol. 7, 132 (2017).

[3] P. Gas, Prz. Elektrotechn. 87, 37 (2011).

[4] D.L. Miller, et al., J. Ultrasound. Med. 31, 623 (2012).

[5] S.B. Devarakonda, et al., Nano Lett. 17, 2532 (2017).

[6] A.P. Sviridov, et al., Appl. Phys. Lett. 103, 193110 (2013).

[7] A. Józefczak, et al., Appl. Phys. Lett. 108, 263701 (2016).

[8] A. Józefczak, et al., J. Magn. Magn. Mater. 431, 172 (2017). 